\title{
CONTROL ORIENTADO A LA HERRAMIENTA PARA DISPOSITIVO ASISTENCIAL EN TAREAS QUE REQUIEREN CAPACIDAD DE PINZADO
}

\author{
Edwin D. Oña, Alberto Jardón, Gabriel Barroso, Carlos Balaguer \\ Robotics Lab, Universidad Carlos III de Madrid, Avda. Universidad 30, Leganés (Madrid), España \\ eona@ing.uc3m.es, ajardon@ing.uc3m.es, gabriel.barroso@airbus.com, balaguer@ing.uc3m.es
}

\section{Resumen}

PRESSMATIC es un dispositivo electromecánico que genera movimientos automáticos de apertura y cierre en su extremo. Está dirigido a personas que conservan movilidad en las extremidades superiores, pero carecen de destreza manual para utilizar herramientas pequeñas como tijeras, cortaúñas o pinzas. En el desarrollo tecnológico del dispositivo, se han utilizado elementos comunes, sin embargo, la aplicación en sí misma es innovadora. En este artículo, se presenta el desarrollo del sistema de control orientado a las herramientas, que ha sido implementado para elevar la usabilidad del dispositivo. Además, se describe brevemente la interfaz gráfica que soporta el control. Se muestran también, los resultados de pruebas con usuarios potenciales y, finalmente se presentan las conclusiones.

Palabras clave: Asistencial, Portátil, Agarre, Electromecánico, Destreza manual

\section{INTRODUCCIÓN}

A día de hoy existen en España y en el resto del mundo millones de personas con algún tipo de diversidad funcional. Según su grado de movilidad, muchas de ellas se encuentran en una situación tal que aun conservando gran parte de la funcionalidad de sus miembros superiores, tienen dificultades para realizar tareas que requieren de cierta destreza manual. Esta situación les puede impedir, entre otras cosas, el uso de determinados objetos de la vida cotidiana para los cuales se necesita un mínimo de destreza. Así, la utilización de herramientas como tijeras, tenazas, cortaúñas, etc. que requiere de acciones muy precisas para su correcta operación, se les puede antojar irrealizable debido a esa falta de movilidad. Puesta de manifiesto esta situación, que a nivel de España se encuentra documentada en un estudio llevado a cabo por el Instituto Nacional de Estadística [6], se contempló la idea de hacerla frente mediante un desarrollo tecnológico que al menos posibilitase a estas personas la utilización de algunas herramientas y por ende les otorgase mayor autonomía en sus actividades básicas de la vida diaria (ABVD).

Las causas por las que una persona carece de movilidad en sus miembros superiores son varias y de naturaleza muy diversa: lesiones, amputaciones, enfermedades, etc. Cualquiera de estas afecciones se traduce en una disminución de las capacidades de la mano de quien las padece, desde la inmovilidad de una sola falange a la imposibilidad de realizar cualquier juego de dedos, muñeca o antebrazo [8]. Para la completa funcionalidad de la mano deben conservarse en buen estado tanto las partes de los aparatos locomotor y circulatorio inherentes a la misma como las capacidades del sistema nervioso encargadas de elaborar, transmitir y controlar los impulsos que intervienen a la hora de desenvolver cualquiera de sus actividades propias. Un fallo o lesión en alguna de esas partes podría derivar en la pérdida de control sobre la musculatura responsable de los movimientos de la mano, que en último término es la ejecutora directa de los mismos. Por otro lado, afecciones óseas (artrosis, artritis, reuma), además de pérdidas mecánicas de movilidad, pueden provocar también dolores tan fuertes que limiten a la persona afectada. Por tanto el abanico de posibles lesiones y de su naturaleza, es extenso. Una mera tendinitis crónica en cualquiera de esos músculos podría suponer una causa de pérdida de movilidad que acarree disfunciones en las actividades de la vida diaria de la persona afectada. Ni que decir tiene que daños en el sistema nervioso (lesiones medulares, enfermedades neurodegenerativas) pueden repercutir en que el individuo pierda parte o la totalidad del control de los músculos mencionados anteriormente.

Esto lógicamente afecta a numerosas actividades de su vida diaria, en mayor o menor medida, dependiendo del alcance de la afección, y provoca situaciones de pérdida de autonomía personal, imposibilidad para continuar desempeñando una actividad laboral, etc.

\subsection{TRABAJOS RELACIONADOS}

La sujeción de herramientas cotidianas, es uno de los principales problemas con los que se encuentran los usuarios a quienes va dirigido este dispo- 


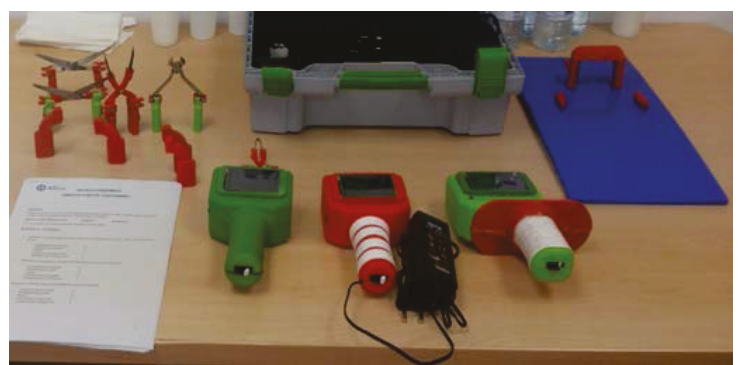

Figura 1: PRESSMATIC con cabezales intercambiables (esquina superior izquierda) y base para intercambio de cabezales (esquina superior derecha)

sitivo. Aunque a día de hoy, encontramos soluciones que facilitan el agarre y sujeción de utensilios [2], son solo adaptaciones [4] y el problema de la falta de control en los movimientos se mantiene, convirtiéndose en una barrera importante.

Por otro lado, se encuentran soluciones basadas en el uso de órtesis. En [3] se propone un mecanismo diferencial de transmisión de potencia basado en cable tensor y muelle. La tensión del cable simula la funcionalidad de un tendón. Para el retorno, se utiliza el resorte para transferir la fuerza del actuador lineal. En [7] se presenta un dispositivo que apoya el movimiento del dedo pulgar e índice. Su sistema también esta basado en actuadores lineales y cables. Por otro lado, en [1] se plantea un exoesqueleto para la mano completa. El diseño del exoesqueleto se basa en anillos de aluminio y también utilizando actuadores lineales.

\subsection{PRESSMATIC: Breve descripción}

El objetivo principal del dispositivo de apoyo PRESSMATIC, es el de asistir a personas que han perdido la capacidad de utilizar con destreza los dedos pulgar e índice (ya sea por una discapacidad, accidente, o por envejecimiento) pero que mantienen cierta capacidad para sujetar objetos, suficiente como para sostener un smartphone. Debido a la falta de destreza en sus dedos, no son capaces por sí mismos de realizar tareas que requieran la capacidad de pinzado. Entre las tareas que resultarían difíciles de realizar para personas con este tipo de problema, encontramos cortar utilizando unas tijeras, recoger o sujetar objetos pequeños, utilizar pinzas, cortaúñas, etc. Poder utilizar alguna de las anteriores herramientas sin PRESSMATIC, conlleva tener cierta destreza y control de movimientos. Por ejemplo, para utilizar unas tijeras hay que introducir los dedos índice y pulgar en los agujeros de la tijera; sujetar unas pinzas de depilar requiere bastante destreza para utilizarlas sin que se caigan; un cortaúñas además de que no se caiga requiere cierta fuerza.

El sistema (Figura 1), consta de tres elementos:

a) Cuerpo principal: donde, a excepción de los cabezales que no son fijos, aloja en su interior todos los elementos funcionales: motor, electrónica de control, mecanismo de conversión de movimiento rotatorio a lineal en el extremo, y pantalla táctil.

b) Cabezales intercambiables: diseñados con la misma funcionalidad que una herramienta de uso común (tijeras, cortaúñas, etc.). Dichos cabezales son intercambiables, y se pueden acoplar al cuerpo principal según la necesidad del usuario en un momento determinado. Así, se busca un dispositivo flexible capaz de realizar varias tareas.

c) Base de ayuda para cambio de cabezales: Para facilitar la tarea de insertar como extraer los cabezales, se ha diseñado un sistema de apoyo para dicha tarea. Para ello, el diseño de los cabezales incorpora salientes laterales además de unos imanes, de manera que se puedan quedar sujetos a la base que también incorpora imanes.

\section{METODOLOGÍA}

Con el fin de conseguir que la manera de utilizar el dispositivo sea lo más fácil, intuitiva y accesible posible de cara al usuario, se implementó un control orientado a la herramienta. Es decir, el dispositivo presenta al usuario las opciones de herramienta que incorpora y ejecuta una tarea preprogramada y adaptada para cada herramienta. Así, el usuario simplemente debe seleccionar la herramienta que mejor se adapte a la tarea que quiera realizar (ver Figura 2).

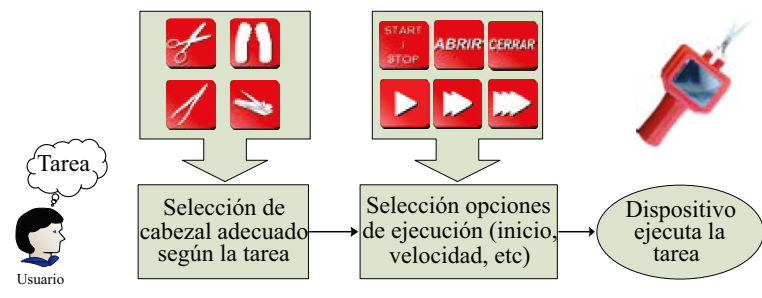

Figura 2: Descripción del control orientado a la herramienta

Para definir las tareas pre-programadas, se hace un análisis de los modos de funcionamiento naturales para cada una de las herramientas, definiendo las especificaciones del control. Como resultado, se definieron tres modos de operación (Tabla 
Tabla 1: Modos de operación y velocidad según herramienta

\begin{tabular}{lcc}
\hline Cabezal & Modo & Velocidad \\
\hline Tijeras & Continuo & Variable \\
Pinzas grandes & Paso a paso & Variable \\
Pinzas pequeñas & Paso a paso & Variable \\
Cortauñas & Simple & Fija \\
\hline
\end{tabular}

1), puesto que las herramientas de pinzas a pesar de ser físicamente diferentes, de cara al control serían iguales.

Del análisis de las herramientas, también se observó que para el uso de las tijeras sería adecuado tener opciones para modificar la velocidad de corte. Así, dentro del modo Continuo se definieron tres niveles de velocidad (rápido, medio y lento). Durante el uso de la herramienta de cortauñas, se observó la necesidad de maximizar el par para el corte. Por lo tanto, para el modo Simple, se ha marcado una velocidad lenta no variable. En el uso de las pinzas no se vio un perfil de funcionamiento restringido. Así, en el modo Paso a paso se habilitan también las tres opciones de velocidad.

\subsection{CONTROL ORIENTADO A LA HERRAMIENTA}

El movimiento lineal en el extremo del dispositivo, se consigue a partir del movimiento rotatorio de un motor paso a paso RS-5350366 y, de por medio, un mecanismo de conversión basado en engranajes y tornillo sinfín. Así, el control sobre el giro del motor, se corresponde con el desplazamiento lineal en el extremo, y por ende, de los cabezales (ver Figura 3).

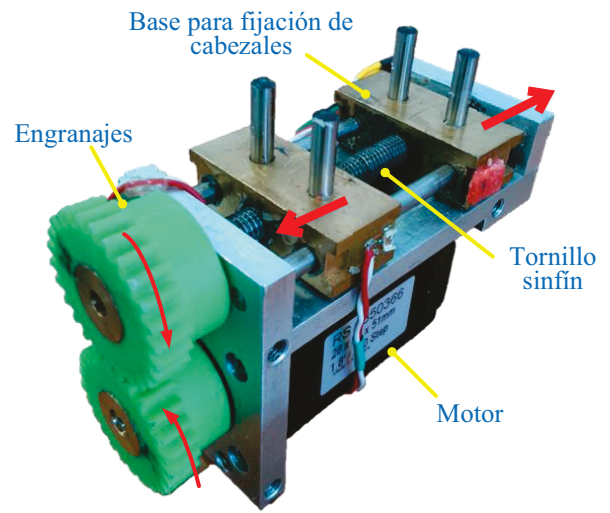

Figura 3: Conversión de movimiento rotatorio a lineal

Se utiliza un driver de potencia Pololu A4988 para alimentar al motor y controlar su movimiento por medio de señales digitales. Para esta aplicación, se han utilizado las señales: Enable (EN), Step (STEP), direction (DIR), y microstepping(MS1, MS2, MS3).

\subsubsection{Modo continuo}

El modo Continuo es el programado para el cabezal de tijeras, y ejecuta ciclos completos de apertura y cierre de forma indefinida. El usuario debe indicar cuando iniciar y cuando detener la ejecución de la tarea.

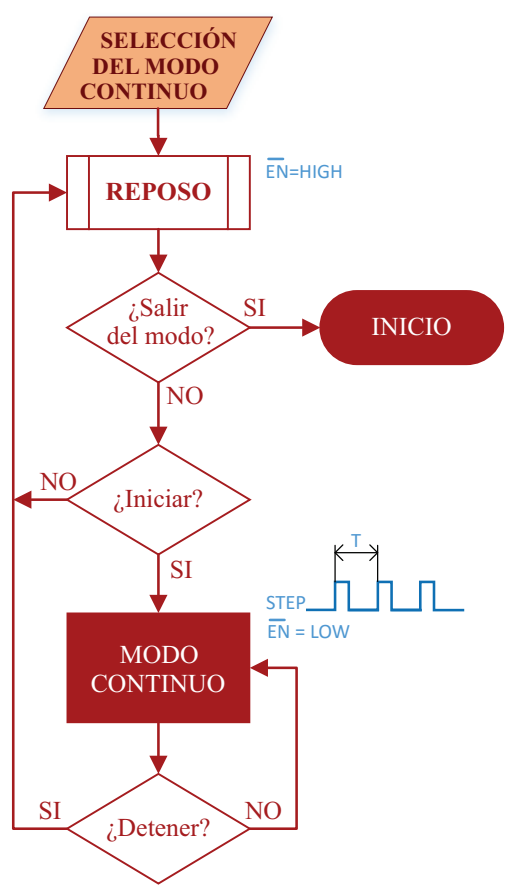

Figura 4: Diagrama de flujo para modo de control 'Continuo'

Éste modo de operación (Figura 4), empieza en un estado de Reposo, donde se deshabilita el movimiento del motor $\left(\mathrm{EN}={ }^{\prime} 1\right.$ ') y donde se mantiene esperando a un cambio de cabezal o a la señal de inicio de cortar. Cuando la señal de iniciar el corte se activa, entra en el modo Continuo donde se habilita el motor $(\mathrm{EN}=$ ' 0 ') y se genera un tren de pulsos de periodo constante. Cuando el usuario active la señal de detener el corte se vuelve al estado de Reposo. Para detectar que se ha alcanzado el recorrido máximo de apertura o cierre, se utilizan los micro interruptores B3U3100P y B3U1100P. Su salida se conecta a dos interrupciones del micro controlador donde se cambia el sentido de giro del motor.

\subsubsection{Modo paso a paso}

El modo paso a paso, es el programado para los cabezales de pinzas y permite realizar movimientos cortos de apertura o cierre, de forma controlada a voluntad del usuario. 


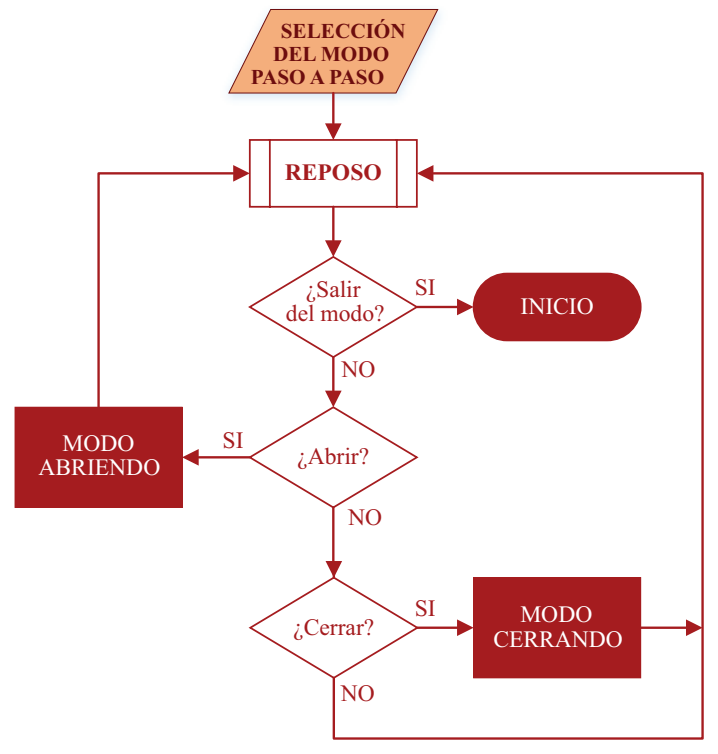

Figura 5: Diagrama de flujo para modo de control 'Paso a paso'

Para conseguir esta funcionalidad (Figura 5), se requieren dos señales de control: una para movimientos de apertura y otra para el cierre. Para permitir al usuario el máximo control sobre los movimientos, se programa que el dispositivo genere movimiento (apertura o cierre), mientras las correspondientes señales de control estén activas. Si el dispositivo alcanza su recorrido máximo de apertura o cierre, el motor se detendrá hasta que se active la señal contraria. Esto se consigue utilizando los interruptores de final de carrera.

\subsubsection{Modo simple}

Finalmente, el modo simple es el utilizado para el cabezal de cortaúñas, y ejecuta únicamente un ciclo completo de apertura y cierre, equivalente al corte de una uña. El usuario volvería a ejecutar un ciclo completo cada vez que esté preparado. En este modo (Figura 6), se limita la apertura del dispositivo a la mitad del recorrido total, suficiente la introducir la uña en la herramienta. Para maximizar la fuerza, no se permite las opciones de cambio de velocidad, y se establece la misma a la más lenta.

\subsection{SISTEMA ELECTRÓNICO}

La programación de los modos de funcionamiento, fue desarrollada siguiendo los diagramas de flujo mostrados anteriormente. El código se implementó bajo la plataforma de código abierto Arduino. Por dicha razón, los elementos hardware empleados han sido compatibles para Arduino.

Para el desarrollo, se utilizó el microcontrolador ATmega2560 que incorpora las placas de desarro-

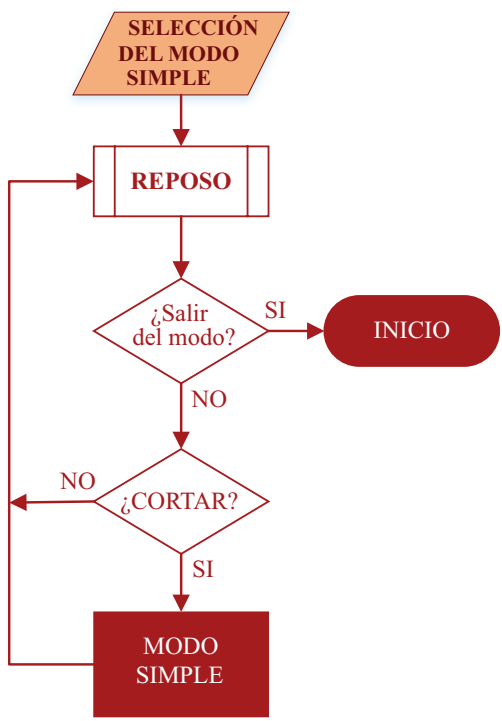

Figura 6: Diagrama de flujo para modo de control 'Simple'

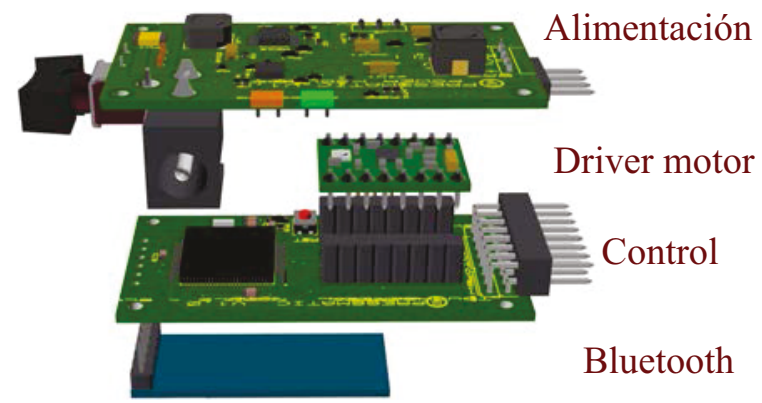

Figura 7: Explosionado de diseño PCB

llo Arduino Mega 2560 y Mega ADK. De los recursos que ofrece dicho microcontrolador, se han utilizado 13 pines para entradas y salidas digitales, una pin de salida analógica para enviar una señal PWM que controle la velocidad del motor, y dos puertos serie, uno a 115200 baudios para comunicación con la pantalla táctil y otro a 9600 baudios que se conecta a un módulo de bluetooth para comunicación con dispositivos externos. Para alimentar el motor, tipo paso a paso bipolar, se utilizó el driver de potencia Pololu A4988 ya que reunía condiciones adecuadas de tamaño, potencia entregada, pocas señales de control y pocos componentes externos adicionales. La velocidad del motor, se ajusta de acuerdo a la frecuencia de una señal PWM que se conecta al driver de potencia, y se traduce en pasos del motor. Además, el A4988 tiene opción de microstepping, lo que permite incrementar su resolución. En nuestro caso, atendiendo a un compromiso entre velocidad de desplazamiento de los cabezales y el par efectivo entregado, se utilizó una frecuencia de $800 \mathrm{~Hz}$ sin que el motor perdiera pasos. 


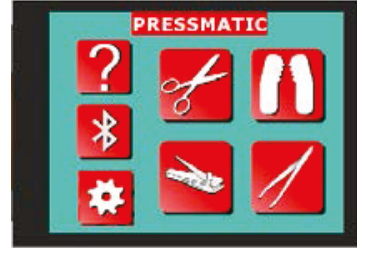

(a)

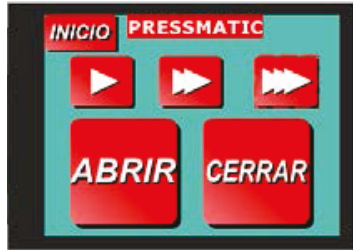

(c)

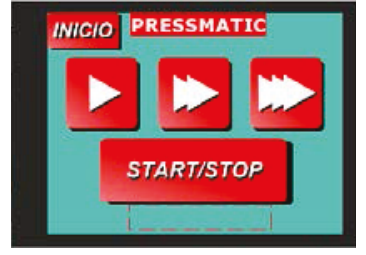

(b)

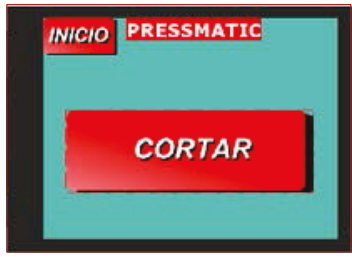

(d)
Figura 8: Interfaz de control para pantalla táctil integrada en color rojo-turquesa. a) menú principal, b) menú control tijeras, c) menú control pinzas, y d) menú control cortaúñas

\subsection{INTERFAZ DE CONTROL}

Para comandar el dispositivo, se requiere una interfaz de control que cumpla con los objetivos de accesibilidad y fácil manejo. Atendiendo a ello, e intentando llegar al mayor número posible de usuarios, se desarrollaron tres canales de comunicación: utilizando una pantalla táctil, una aplicación Android para smartphone y control por comandos de voz.

\subsubsection{Pantalla táctil}

El primer canal de comunicación, utiliza una interfaz gráfica implementada en una pantalla táctil integrada en el cuerpo principal. Se ha elegido la pantalla resistiva modelo uLCD-28PTU [11] ya que ofrece un tamaño de 2.8 pulgadas, adecuado para nuestra aplicación, un sencillo entorno de programación gráfico y comunicación por puerto serie. Aunque por sensibilidad al tacto, una pantalla capacitiva es una mejor opción se optó por la actual pantalla resistiva puesto que el objetivo del prototipo es validar la funcionalidad del mismo y la utilidad de la pantalla integrada. A través de la pantalla táctil, se presentan de forma visual las opciones de herramienta al usuario. Se utilizan pictogramas, diseñados para cada herramienta, con la intención de hacer el uso de PRESSMATIC más intuitivo. En la Figura 8, se muestran la interfaz gráfica diseñada. En el diseño de la interfaz gráfica, se han tenido en cuenta criterios de accesibilidad de páginas web para permitir una mejor visibilidad de los iconos y poder reconocer su función de una manera sencilla.

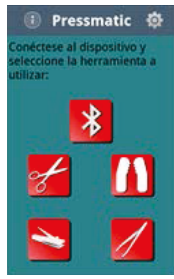

(a)

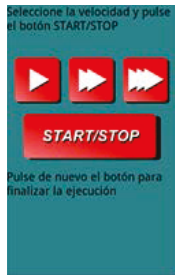

(b)

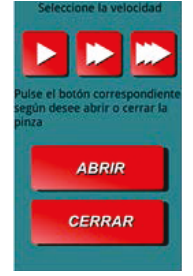

(c)

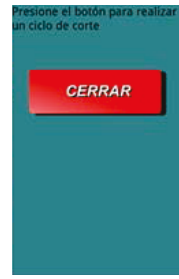

(d)
Figura 9: Menús de navegación para aplicación Android: a) menú principal, b) menú control tijeras, c) menú control pinzas, y d) menú control cortaúñas

\subsubsection{Aplicación android para móvil}

El segundo canal de comunicación es a través de una aplicación Android que, instalada en un smartphone, puede vincularse a PRESSMATIC mediante bluetooth. El OS Android es una plataforma de código abierto y software libre, que cuenta con una comunidad de desarrolladores muy competente como por ejemplo Stackoverflow [9], y ocupa la mayoría de cuota de mercado estando instalado en más del $80 \%$ de los terminales en el mundo [5].

En el desarrollo de la aplicación para móvil, se ha mantenido el diseño gráfico implementado en la pantalla táctil, es decir, se han utilizado los mismos pictogramas, mismo menús de navegación, mismos colores, y sobre todo la misma funcionalidad. Además, se han tenido en cuenta los criterios de accesibilidad recogidos en [10], [2]. En la Figura 9, se muestran los menús implementados en la aplicación móvil, y que se corresponden con los menús desarrollados para la pantalla táctil (anterior Figura 8).

Para comunicar el smartphone con PRESSMATIC, la electrónica de control abordo incluye un emisor-receptor bluetooth HC-05 compatible con Arduino. Desde la aplicación móvil se envían comandos predefinidos correspondientes a una acción de pulsar o selección en el menú. El módulo HC-05 recibe los comandos y los envía por puerto serie al microcontrolador que ejecuta la tarea correspondiente.

En la Tabla 2, se muestra los comandos utilizados y las acciones a las que se corresponden, agrupados de acuerdo a la pantalla en la que se encuentran. Para mostrar el funcionamiento, planteamos un ejemplo de un usuario que quiere utilizar el cabezal de tijeras a una velocidad intermedia. Desde la pantalla de inicio (Figura 9-a), que se corresponde con el grupo 'Selección de herramientas', se seleccionan las tijeras, la aplicación móvil envía el comando 'b' correspondiente al modo continuo. 
Tabla 2: Comandos protocolo bluetooth

\begin{tabular}{llc}
\hline Grupo & Menú/Acción & Comando \\
\hline \multirow{2}{*}{ Selección de } & Modo continuo & $\mathrm{b}$ \\
herramientas & Modo Paso a paso & $\mathrm{c}$ \\
& Menu Bluetooth & $\mathrm{e}$ \\
\hline \multirow{3}{*}{ Menú de } & Velocidad baja & 5 \\
tijeras & Velocidad media & 6 \\
& Velocidad rápida & 7 \\
& Inicio-Parada & 0 \\
\hline \multirow{2}{*}{ Menu grip } & Abrir & 1 \\
& Cerrar & 2 \\
\hline Menu de & Reposo & $\mathrm{z}$ \\
corta uñas & Un corte & 8 \\
\hline Menu de & Conectar-Desconectar & 9 \\
bluetooth & Atrás-Inicio & $\mathrm{a}$ \\
\hline Común & & \\
\hline
\end{tabular}

La aplicación móvil cambia automáticamente de pantalla y presenta el menú correspondiente a las tijeras (Figura 9-b), donde tenemos disponibles los botones de velocidad y de inicio/parada.

$\mathrm{Al}$ establecer la velocidad intermedia, la aplicación móvil envía el comando ' 6 ' que establece dicha velocidad en PRESSMATIC. Finalmente, al pulsar el botón de inicio, se enviaría el comando ' 0 ' para empezar a cortar. Los comandos definidos en la Tabla 2, no contienen información por sí mismos, sino que ayudan a la electrónica de control, a distinguir una acción de pulsar un botón y a que botón se corresponde, permitiéndole actuar en consecuencia.

Esta segunda interfaz gráfica presenta algunas ventajas respecto a la pantalla integrada. Por un lado, puede funcionar de forma simultánea con la pantalla táctil, sin interferir la una con la otra. Cada cambio o acción en una interfaz, se refleja automáticamente en la otra. Así, a través del móvil, permite a los usuarios controlar el dispositivo como si se tratase de una pantalla remota. Por otro lado, se instala en el smartphone del usuario final, con el que se va a encontrar mucho más familiarizado pudiendo manejarlo de forma más fluida y cómoda. Además, la pantalla capacitiva del smartphone, mejora notoriamente la sensibilidad táctil de la pantalla resistiva integrada, disminuyendo la dificultad de uso.

\subsubsection{Comandos de voz}

Finalmente, el tercer canal de comunicación es mediante comandos de voz. Para ello, se utilizó el módulo de reconocimiento de voz EasyVR 2.0, que es capaz de reconocer palabras pregrabadas en su memoria y enviar un comando definido para cada palabra a través de su puerto serie. Los comandos enviados, son los mismos que utiliza la aplicación móvil para mantener la coherencia. Para implementar el control por voz, se conectó el módulo EasyVR a un Arduino Mini y un módulo bluetooth HC-05. El Arduino Mini, servía como una pasarela que permite envíar los comandos reconocidos por el módulo de voz, a través del dispositivo bluetooth. Esta última forma de controlar el dispositivo, no ha podido ser desarrollada ampliamente, pero ofrece una vía muy a tener en cuenta para futuras iteraciones.

\section{RESULTADOS}

El funcionamiento del control propuesto, en primer lugar se valida de forma satisfactoria en el laboratorio, comprobando que la integración entre los distintos canales de comunicación, así como el control del dispositivo no presentan ningún problema. Para evaluar la funcionalidad del control por parte de un usuario potencial, se propuso realizar varias tareas a un individuos con lesión medular. Los participantes carecen de destreza manual y no son capaces por sí solos de utilizar unas tijeras, ni manipular objetos pequeños.

Se plantea realizar dos tareas utilizando únicamente PRESSMATIC y el cabezal adecuado para llevarlas a cabo. La primera tarea propuesta consiste en cortar varias figuras geométricas impresas sobre un folio de papel tamaño A4. En la Figura 10 se muestra a uno de los individuos realizando la tarea.

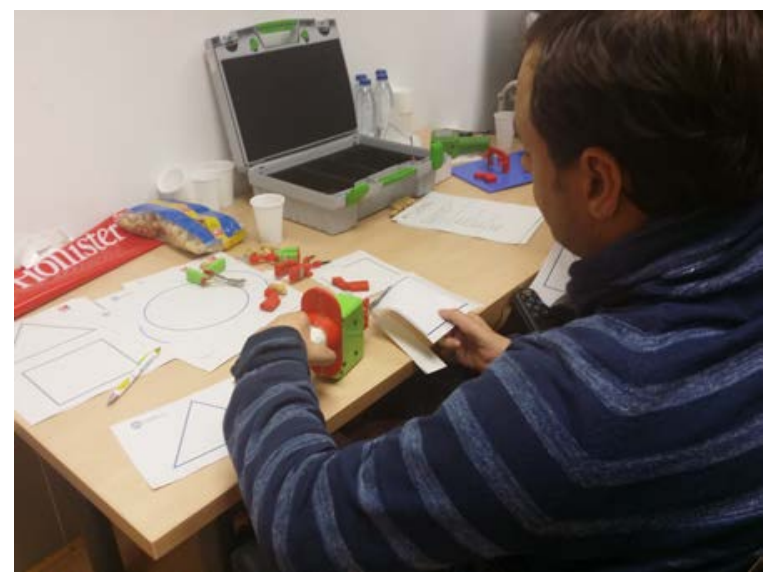

Figura 10: Sujeto realizando tarea de corte

La segunda tarea consiste en recoger objetos pequeños y colocarlos en un recipiente. Entre los objetos a recoger tenemos los cubos $(25 \mathrm{~mm}$ de arista) de un test ampliamente utilizado en la evaluación de destreza y coordinación manual, denomi- 
nado Box \& Blocks Test. Otro de los objetos fue una memoria UBS Pendrive. En la Figura 11 se muestra a otro participante realizando la segunda tarea.

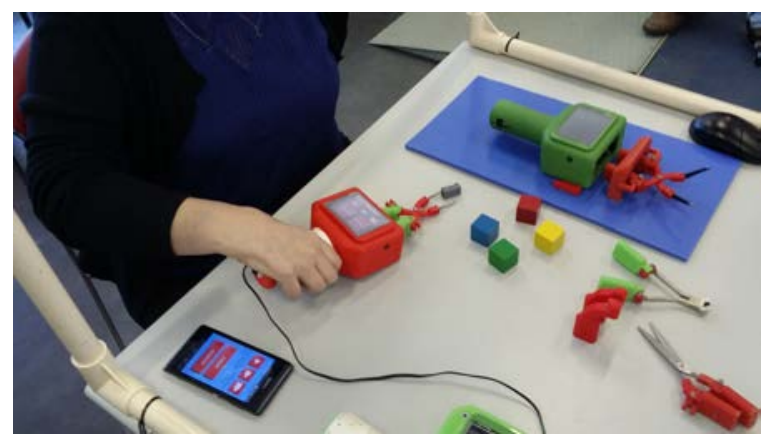

Figura 11: Sujeto realizando tarea de recoger objeto pequeño

\section{CONCLUSIONES}

En este trabajo, se presenta el diseño e implementación del control de un dispositivo asistencial para tareas que requieren de destreza manual. Para facilitar la usabilidad del dispositivo, se desarrolla un control orientado a la herramienta. A tal fin, se define un set de herramientas que incluyen tijeras, pinzas grandes y pequeñas, y cortauñas. Tras analizar la el modo de funcionamiento de cada herramienta, se definen tres modos de operación que se pre-programan de cara al usuario. Para validar el control propuesto, se lleva el dispositivo a un centro de cuidado de personas con discapacidad. Se propone el desarrollo de dos tareas: cortar figuras geométricas de un folio, y recoger objetos pequeños. Los participantes, presentan varios niveles de lesión medular, careciendo de destreza manual para realizar las tareas propuestas por sí solos. Los resultados son satisfactorios puesto que todos los participantes son capaces de completar las tareas, en mayor o menor tiempo. Además, a partir de la experiencia de usuarios potenciales se valida el control del dispositivo, teniendo mayor acogida el uso de las herramientas por medio de la app instalada en un smartphone.

\section{Agradecimientos}

A la Fundación Caser por el premio en I+D, a la Fundación Universia por financiar este proyecto, y al equipo del Robotics Lab. La investigación que lleva a estos resultados ha recibido financiación del proyecto RoboCity2030-III-CM (Robótica aplicada a la Mejora de la Calidad de Vida de los Ciudadanos Fase III; S2013/MIT-2748), financiado por Programas de Actividades I+D en la Comunidad de Madrid y cofinanciado por los Fondos Estructurales de la UE.

\section{Referencias}

[1] Baker, M. D., McDonough, M. K., McMullin, E. M., Swift, M., and BuSha, B. F. (2011, April). Orthotic hand-assistive exoskeleton. In 2011 IEEE 37th Annual Northeast Bioengineering Conference (NEBEC) (pp. 1-2). IEEE.

[2] CEAPAT (2006).Catálogo de Ayudas técnicas, Centro Estatal de Autonomía Personal y Ayudas Técnicas, centro tecnológico dependiente del IMSERSO, Ministerio de Trabajo y Asuntos Sociales. http://www. ceapat.es/

[3] Goutam, S., and Aw, K. C. (2014, September). Development of a compliant hand assistive device. In Mechatronic and Embedded Systems and Applications (MESA), 2014 IEEE/ASME 10th International Conference on (pp. 1-6). IEEE.

[4] Hesse, S., Bardeleben, A., Werner, C., \& Kirker, S. (2011). Magnetic Grip Facilitates Feeding With Weakened Hands After Spinal Cord Injury. Neurorehabilitation and neural repair.

[5] IDC (2014). International Data Corporation. urlhttp://www.idc.com/prodserv/smartphoneos-market-share.jsp

[6] INE (2008). Encuesta de Discapacidad, Autonomía personal y situaciones de Dependencia (EDAD). Instituto nacional de estadística.

[7] Makhdoomi, M. R., Hamid, A. M. B., and Saleh, T. (2015, May). Development and performance evaluation of a linear actuator based wearable assistive device. In Control Conference (ASCC), 2015 10th Asian (pp. 1-6). IEEE.

[8] OMS, Banco Mundial (2011). Informe Mundial sobre Discapacidad.

[9] Stackoverflow (2000). Comunidad de desarrolladores. www.stackoverflow.com

[10] VVAA (2009). Comunidad de desarrolladores de Android. http://developer.android. com

[11] 4DSYSTEMS (2001). http://www. 4dsystems.com.au/ 